Dedicated to Masaki Kashiwara, a great master of harmonic analysis, on the occasion of his 70th birthday

\title{
AFFINE HECKE ALGEBRAS VIA DAHA
}

\author{
IVAN CHEREDNIK ${ }^{\dagger}$
}

This paper is based on the lecture delivered at the conference "Algebraic Analysis and Representation Theory" in honor of Masaki Kashiwara's 70th birthday, an author's prior talk at MIT and his course at UNC Chapel Hill. It is aimed at obtaining the Plancherel measure for the regular representation of Affine Hecke Algebras (AHA) as the limit $q \rightarrow 0$ of the integral-type formulas for the DAHA inner products in the polynomial and related modules. The usual integral formulas generally serve only $\Re k>0$ (in the DAHA parameters $t=q^{k}$ ) and must be analytically continued to negative $\Re k$, which is a $q$-generalization of "picking up residues" due to Arthur, Heckman, Opdam and others (can be traced back to Hermann Weyl). When this is done, we arrive at finite sums of integrals over double affine residual subtori, though the full procedure is known only in type $A$ by now. This is not related to the DAHA reducibility of the polynomial and similar DAHA modules. The formulas are nontrivial for any $\Re k<0$, not only for singular $k<0$ resulting in the DAHA reducibility. For singular $k$ in type $A$, they provide the decomposition of the polynomial representation in terms of the irreducible modules. As we demonstrate, this is quite interesting even for $A_{1}$.

The decomposition of the regular AHA representation in terms of (unitary) irreducible modules is an important part of algebraic harmonic analysis, involving deep geometric methods (Kazhdan- Lusztig and others). As an expected application, our approach would allow to interpret formal degrees of AHA discrete series via DAHA, without any geometry. Paper ${ }^{21]}$ do this within the AHA theory, but the DAHA level is expected to be quite clarifying and more powerful (with an additional parameter $q$ ).

We mainly discuss the spherical case and provide explicit analytic continuations only for $A_{1}$. The key is that the mere uniqueness of the DAHA inner product fixes uniquely the $q$-generalization of the corresponding Arthur-Heckman-Opdam formula, including very interesting $q$-counterparts of formal degrees.

Even in the spherical case, the procedure of analytic continuation to $\Re(k)<0$ is technically involved. There are no significant theoretical challenges here, but practical finding double affine residual subtori

† Partially supported by NSF grant DMS-1363138. 
and their contributions to the DAHA inner products is performed (partially) only for $A_{n}$ at the moment. The passage to the whole regular representation will presumably require the technique of hyperspinors, which we outline a bit at the end of this paper.

Importantly, there is no canonical AHA-type trace in the DAHA theory; instead, we have the theory of DAHA coinvariants serving DAHA anti-involutions. There are of course other aspects of DAHA harmonic analysis: the unitary dual, calculating Fourier transforms of DAHA modules and so on, where the regular representation of DAHA must be studied (by analogy with the AHA theory). However, we focus on the spherical part of the regular AHA representation, which becomes an irreducible module in the DAHA theory.

Only basic references are provided in the paper; see there for further information. Also, the general AHA and DAHA theory is quite compressed. Full details are provided for $A_{1}$; the generalization to $A_{n}$ follows the same lines.

We do not give general definitions (for arbitrary root systems) in this note. These definitions, including the basic features of DAHA inner products, are (published and) sufficiently well known for $\Re(k)>0$; the main references are ${ }^{[2,3,8]}$. The extension of the corresponding integral formulas to $\Re(k)<0$ is the aim of this work; we think that the case of $A_{1}$ gives a clear direction. Importantly, only relatively elementary tools from "q-calculus" are needed here. DAHA theory guarantees that the inner products under consideration do have analytic and meromorphic continuations, but does not provide explicit formulas (summations of integrals over generalized residual subtori). This is actually similar to $[15,21]$. The sections of the paper are:

1. On Fourier Analysis

3. Shapovalov pairs

5. General DAHA $\left(A_{1}\right)$

7. $\mathrm{P}$-adic limit
2. AHA-decomposition

4. Rational DAHA $\left(A_{1}\right)$

6. Analytic continuation

8. Conclusion.

The following table sketches the basic "levels" in harmonic analysis on AHA vs. those in the corresponding DAHA theory. We think it explains our take on the AHA theory sufficiently well. The second column here is technically adding an extra parameter $q$ to the theory. Conceptually, the passage to DAHA provides important rigidity, which is of clear importance for the AHA Plancherel formula and related problems. The key is that Fourier transform is essentially an involution in the DAHA theory, which is so different from AHA theory and HarishChandra theory.

† Partially supported by NSF grant DMS-1363138 


\begin{tabular}{|c|c|}
\hline HA on AHA & HA on DAHA \\
\hline Unitary(spherical) dual & Polynomial/induced modules \\
\hline AHA Fourier transform & $\mathcal{H}-$ - automorphism $Y \rightarrow X^{-1}$ \\
\hline Trace formulas, $L^{2}(\mathcal{H})$ & Inner products as integrals \\
\hline
\end{tabular}

The three "stages" in the first column are common in harmonic analysis on symmetric spaces and related/similar theories. One can ask the same questions for DAHA. The first two stages are meaningful; DAHA provides an important source of new infinite-dimensional unitary theories, which are of great demand in analysis and physics. However, our understanding is that there is no canonical DAHA trace; accordingly, it is not clear what the theory of $L^{2}(\mathcal{H})$ can be. Instead, we have the analytic theory of DAHA involutions and coinvariants. The key for us is the interpretation of the spherical part of the regular AHA representation as DAHA polynomial representation, with very rich structures.

One of possible applications of this program can be a new approach to formal degrees of AHA discrete series via DAHA. Let us mention (at least) Kazhdan, Lusztig, Reeder, Shoji, Opdam, Ciubotaru, S.Kato in this regard; see some references below.

\section{On Fourier Analysis}

This section is mainly needed to put this work into perspective. The classical Fourier Transform, $\mathrm{FT}=\int e^{2 \lambda x}\{\cdot\} d x$, is naturally associated with the automorphism $x \rightarrow y=d / d x \rightarrow-x$ of the $d=1$ Heisenberg algebra. This can be readily extended to any dimensions $d$ and to any root systems. Its spherical generalization is a famous Harish-Chandra transform, but this is beyond the Heisenberg algebras. Classically, the Fourier transform can be related to $\left(\begin{array}{cc}0 & 1 \\ -1 & 0\end{array}\right) \in S L_{2}$, though this kind of interpretation seems a special feature of $d=1\left(A_{1}\right)$; see below. Similarly, the Weyl algebra at $q=e^{\frac{2 \pi i}{N}}$ can be used to study $F_{N}=\sum_{j=0}^{N-1} q^{\lambda j}\{\cdot\}$, which can be readily generalized to any $d$. Finding counterparts of Heisenberg/Weyl algebras directly serving the HarishChandra transform and its variants and generalizations is a natural question here. DAHA essentially manages this. Using Lie groups here is generally insufficient even for the classical one-dimensional hypergeometric function.

\section{Some famous challenges.}

Problem 1. Extending Lie theory from spherical functions to hypergeometric functions (in any ranks), the Gelfand Program. Here KacMoody algebras (conformal blocks, to be more exact) and Lie supergroups can be used, but the problem still appeared beyond Lie theory. 
Problem 2. Can Fourier transform be interpreted as a reflection in the Weyl group (in any rank)? Unlikely so. Say, there are 3 candidates (reflections) for FT in $S L_{3}$, but it can be expected unique due to the key property of FT in any theories: they send polynomials to $\delta$-functions.

Problem 3. A counterpart of $\mathrm{FT}\left(e^{-x^{2}}\right)=\sqrt{\pi} e^{+\lambda^{2}}$ at roots of unity is the formula $F_{N}\left(q^{j^{2}}\right)=\zeta \sqrt{N} q^{-\lambda^{2}}$ for $\zeta \in\{0,1, \imath, 1+\imath\}$. The Weyl algebra gives $\sqrt{N}$ but does not catch $\zeta$, i.e. it provides only the absolute values of the Gauss sums. Can this be improved?

DAHA approach. Concerning Problems 1-2, the reproducing kernel of the DAHA-Fourier transform (its square is essentially one) is the generalized "global" difference hypergeometric function; any root systems were managed. Problem 3 can be settled too (within the theory of DAHA-Gauss-Selberg sums). The question we (partially) address in this paper is, what do these developments give for AHA?

Before coming to this, let us quickly discuss global hypergeometric functions, which seem the main application of DAHA with known and expected applications well beyond harmonic analysis. In the $p$-adic limit, which is $q \rightarrow 0$, they reduce to the polynomials; classical hypergeometric functions are for $q \rightarrow 1$.

Global functions $\Phi_{q, t}(X, \Lambda), q<1 .{ }^{[3]}$ These functions are defined as reproducing kernels of the DAHA Fourier Transform; no difference equations are used in this approach for their definition. To calculate them we use that this transform sends Laurent polynomials in terms of $X=q^{x}$ times the Gaussian $q^{-x^{2} / 2}$ to such polynomials times $q^{+x^{2} / 2}$, which is similar to theory of Hankel transform. This gives an explicit formula for the series $\widetilde{\Phi}_{q, t}(X, \Lambda) \stackrel{\text { def }}{=} \theta_{R}(X) \theta_{R}(\Lambda) \Phi_{q, t}(X, \Lambda) / \theta_{R}\left(t^{\rho}\right)$ in terms of $P_{\mu}(X) P_{\mu}(\Lambda)$ for Macdonald polynomials $P_{\mu}$.

This is general theory, for any (reduced, irreducible) root systems $R$. The Laurent polynomials are in terms of $X_{\lambda}=q^{(x, \lambda)}$ for $\lambda \in$ $P$ (the weight lattice for $R$ ),$(\cdot, \cdot)$ is the standard $W$-invariant inner product, $x^{2}=(x, x), \theta_{R}$ is the usual theta-series associated with $R$, $\mu \in P$. This is from ${ }^{[3]}$; see also ${ }^{[2,5]}$. The series $\widetilde{\Phi}_{q, t}(X, \Lambda)$ is absolutely convergent as $|q|<1, W$-invariant with respect to both, $X$ and $\Lambda$, and, importantly, $X \leftrightarrow \Lambda$-symmetric (as for the Bessel functions). Here one must avoid the poles of the coefficients of Macdonald polynomials and zeros of $\theta_{R}(X)$; otherwise the convergence is really "global". Such global functions are missing in the (differential) Harish-Chandra theory.

Furthermore, let $X=q^{x}, \Lambda=q^{\lambda}$. Assume that $\lambda=w\left(\lambda_{+}\right)$for dominant $\lambda_{+}$such that $\Re\left(\lambda_{+}, \alpha_{i}\right)>0$, i.e. that $\lambda$ is generic. Then $\Phi_{q, t}(X, \Lambda)$ under some explicit normalization becomes an asymptotic series $\Phi_{q, t}^{a s}(X, \Lambda)=q^{-\left(x, \lambda_{+}\right)} t^{\left(x+\lambda_{+}, \rho\right)}(1+\ldots)$ as $\Re\left(x, \alpha_{i}\right) \rightarrow+\infty$. 
Harish-Chandra decomposition. See I.Ch ${ }^{[5,9]}$, J.Stokman ${ }^{[19]}$. It is:

$$
\Phi_{q, t}(X, \Lambda)=\sum_{w \in W} \sigma_{q, t}(w(\Lambda)) \Phi_{q, t}^{a s}(X, w(\Lambda))
$$

for the $q$,t-extension $\sigma_{q, t}(\Lambda)$ of the Harish-Chandra $c$-function. Note that (naturally) $\mathcal{L}_{p} \Phi_{q, t}(X, \Lambda)=p(\Lambda) \Phi_{q, t}(X, \Lambda)$ for $p \in \mathbb{C}[X]^{W}$ and Macdonald-Ruijsenaars operators $\mathcal{L}_{p}$ in type $A$ (and due to DAHA for any root systems). This relation is not used in the definition of $\Phi_{q, t}$. The recovery formula is more important here: $P_{\lambda}$ is proportional to $\Phi_{q, t}(X, \Lambda)$ for $\Lambda=t^{\rho} q^{\lambda}$ (with an explict coefficient of proportionality).

Let us give the exact formulas for $A_{1}$. For Rogers-Macdonald polynomials $P_{n}(X), \mu$ provided below, and $\theta(X) \stackrel{\text { def }}{=} \sum_{m=-\infty}^{\infty} q^{m x+m^{2} / 4}$,

$$
\frac{\theta(X) \theta(\Lambda)}{\theta\left(t^{1 / 2}\right)} \Phi=\widetilde{\Phi}_{q, t}(X, \Lambda) \stackrel{\text { def }}{=} \sum_{n=0}^{\infty} q^{\frac{n^{2}}{4}} t^{\frac{n}{2}} \frac{P_{n}(X) P_{n}(\Lambda)(\mu)_{\mathrm{CT}}}{\left(P_{n} P_{n} \mu\right)_{\mathrm{CT}}},|q|<1 .
$$

For $|X|<|t|^{\frac{1}{2}}|q|^{-\frac{1}{2}}$, the Harish-Chandra formula reads: $\widetilde{\Phi}_{q, t}(X, \Lambda)=$

$$
\begin{aligned}
& =(\mu)_{\mathrm{CT}} \sigma(\Lambda) \theta\left(X \Lambda t^{-1 / 2}\right) \sum_{j=0}^{\infty}\left(\frac{q}{t}\right)^{j} X^{2 j} \prod_{s=1}^{j} \frac{\left(1-t q^{s-1}\right)\left(1-q^{s-1} t \Lambda^{-2}\right)}{\left(1-q^{s}\right)\left(1-q^{s} \Lambda^{-2}\right)} \\
& +(\mu)_{\mathrm{CT}} \sigma\left(\Lambda^{-1}\right) \theta\left(X \Lambda^{-1} t^{-1 / 2}\right) \sum_{j=0}^{\infty}\left(\frac{q}{t}\right)^{j} X^{2 j} \prod_{s=1}^{j} \frac{\left(1-t q^{s-1}\right)\left(1-q^{s-1} t \Lambda^{2}\right)}{\left(1-q^{s}\right)\left(1-q^{s} \Lambda^{2}\right)}
\end{aligned}
$$

where $\sigma(\Lambda)=\prod_{j=0}^{\infty} \frac{1-t q^{j} \Lambda^{2}}{1-q^{j} \Lambda^{2}}$ is the $q$, t-generalization of the HarishChandra $c$-function; $(\cdot)_{\text {Ст }}$ is the constant term. See ${ }^{[9]}$. The sums here are nothing but (special) Heine's basic hypergeometric functions. Letting here $t \rightarrow 0$ in type $A_{n}$, the asymptotic expansions of the resulting global q-Whittaker function are essentially the Givental-Lee functions. This is an important connection between the physics $B$-model (the usage of the global function) and the $A$-model (the usage of its asymptotic expansions). We note that $\Phi$ is actually an entirely algebraic object, uniquely determined by its asymptotic behavior, including the walls (resonances), when $\Re(\alpha, \lambda)=0$ for some roots $\alpha$ (the theory of resonances is still incomplete).

\section{AHA-DECOMPOSITION}

Let $R \subset \mathbb{R}^{n}$ be a root system, $Q \subset P$ (the weight lattice), $W=\left\langle s_{\alpha}\right\rangle$ for $\alpha \in R, \widetilde{W}=W \ltimes Q \subset \widehat{W}=W \ltimes P=\widetilde{W} \ltimes \Pi$, where $\Pi=P / Q$.

Then $\mathcal{H} \stackrel{\text { def }}{=}\left\langle\Pi, T_{i}(0 \leq i \leq n)\right\rangle /\{$ homogeneous Coxeter relations for $T_{i}$, and $\left(T_{i}-t^{\frac{1}{2}}\right)\left(T_{i}+t^{-\frac{1}{2}}\right)=0$ for $\left.1 \leq i \leq n\right\}$, where $\mathbb{R}$ will be the ring of coefficients, including $q, t^{ \pm 1 / 2}$. This is convenient to avoid the complex conjugation in the scalar products (and for positivity). 
We set $T_{\widehat{w}}=\pi T_{i_{l}} \cdots T_{i_{1}}$ for reduced decompositions $\widehat{w}=\pi s_{i_{l}} \cdots s_{i_{1}} \in \widehat{W}$, where $l=l(\widehat{w})$ is the length of $\widehat{w}$. The canonical anti-involution, trace and scalar product are:

$$
T_{\widehat{w}}^{\star} \stackrel{\text { def }}{=} T_{\widehat{w}^{-1}},\left\langle T_{\widehat{w}}\right\rangle=\delta_{i d, \widehat{w}},\langle f, g\rangle \stackrel{\text { def }}{=}\left\langle f^{\star} g\right\rangle=\sum_{\widehat{w} \in \widehat{W}} c_{\widehat{w}} d_{\widehat{w}},
$$

where $f=\sum c_{\widehat{w}} T_{\widehat{w}}, g=\sum d_{\widehat{w}} T_{\widehat{w}} \in L^{2}(\mathcal{H})=\left\{f, c_{\widehat{w}} \in \mathbb{R}, \sum c_{\widehat{w}}^{2}<\infty\right\}$.

According to Dixmier, $\langle f, g\rangle=\int_{\pi \in \mathcal{H}^{\vee}} \operatorname{Tr}\left(\pi\left(f^{\star} g\right)\right) d \nu(\pi)$. We omit here some analytic details concerning the classes of functions. In the spherical case (referred to as "sph" later on), one takes $f, g \in P_{+} \mathcal{H} P_{+}$, where $P_{+} \stackrel{\text { def }}{=} \sum_{w \in W} t^{\frac{l(w)}{2}} T_{w}$. The measure reduces correspondingly.

Macdonald found an integral formula for $\nu_{\text {sph }}(\pi)$, as $t>1$. Its extension to $0<t<1$ (due to ... Arthur, Heckman-Opdam, ...) by the analyticity is sometimes called "picking up residues" $[12,15,21,23]$. The final formula (for any $t$ ) generally reads:

$$
\int\{\cdot\} d \nu_{s p h}^{a n}(\pi)=\sum C_{s, S} \cdot \int_{s+i S}\{\cdot\} d \nu_{s, S},
$$

summed over (affine) residual subtori $s+S$. Residual points (very interesting and the most difficult to reach) correspond to square integrable irreducible modules (as their characters $\chi_{\pi}$ extend to $L^{2}(\mathcal{H})$ ).

This formula involves deep algebraic geometry, the Kazhdan-Lusztig theory ${ }^{[17,18]}$. In our approach via DAHA, this very formula expected to be a reduction of the analytic continuation of the DAHA inner product in the integral form, which requires only $q$-calculus. The main claim is as follows. It is in the spherical case and is a theorem for any (reduced, irreducible) root systems, with an important reservation that the explicit formula is known by now only in type $A$ (unpublished).

The q,t-generalization of the picking up residues is the presentation of the inner product in the DAHA polynomial representation as sum of integrals over DAHA residual subtori. Only the whole sum satisfies the DAHA invariance, and the corresponding $C$-coefficients are uniquely determined by this property. Upon the limit $q \rightarrow 0$, this approach potentially provides explicit formulas for the $C_{s, C^{-}}$-coefficients above, including formal degrees (for the residual points).

\section{Shapovalov Pairs ${ }^{[8]}$}

We will now switch to the DAHA harmonic analysis. In contrast to the Harish-Chandra theory, where we mainly have two theories based on the imaginary and real integration, the so-called compact and noncompact cases, here we have more options. Let us try to outline them, disregarding various (many) specializations and the open project aimed 
at the passage from the $q$-Gamma function in DAHA theory to the $p$ adic Gamma (this is doable, but there are no works on this so far).

We think that there are essentially 6 major theories by now, corresponding to different choices of "integrations"; some connections are shown by arrows. We stick to the imaginary integration in this paper.

DAHA INTEGRATIONS:

\begin{tabular}{|c||c|}
\hline imaginary $(|q| \neq 1)$ & real $(|q| \neq 1)$ \\
$\Downarrow$ & $\Downarrow$ \\
constant term $(\forall q)$ & Jackson sums \\
$\Uparrow$ & $\Downarrow$ \\
the case $|q|=1 \Rightarrow$ & $\Rightarrow$ roots of unity \\
\hline
\end{tabular}

As above, $R \subset \mathbb{R}^{n}$ is a root system (irreducible and reduced), $W$ denotes the Weyl group $<s_{i}, 1 \leq i \leq n>, P$ is the weight lattice.

We omit the general definition of DAHA (it will be provided later for $\left.A_{1}\right)$; see ${ }^{[2]}$. The following will be sufficient. For $T_{w}$ as above,

$$
\mathcal{H}=\left\langle X_{b}, T_{w}, Y_{b}, q, t\right\rangle, b \in P, w \in W, \mathbb{R} \ni t^{ \pm 1 / 2}, q=\exp \left(-\frac{1}{a}\right), a>0,
$$

where the ring of coefficients is $\mathbb{R}$. More formally, it is defined over $\mathbb{Z}\left[q^{ \pm \frac{1}{m}}, t^{ \pm \frac{1}{m}}\right]$ for proper $m$.

Definition 3.1. The Shapovalov anti-involution $\varkappa$ of $\mathcal{H}$ for $Y$ is such that $T_{w}^{\varkappa}=T_{w^{-1}}$ and the following "PBW property" holds: for any $H \in$ $\mathcal{H}$, the decomposition $H=\sum c_{a w b} Y_{a}^{\varkappa} T_{w} Y_{b}$ exists and is unique.

An example. Let $\varkappa: X_{b} \leftrightarrow Y_{b}^{-1}, T_{w} \rightarrow T_{w^{-1}}(w \in W)$. All Macdonald conjectures follow from its mere existence (without using the shift operator, and practically without any calculations); see ${ }^{[4]}$.

Definition 3.2. The coinvariant is $\{H\} \varrho_{\varkappa} \stackrel{\text { def }}{=} \sum c_{a w b} \varrho\left(Y_{a}\right) \varrho\left(T_{w}\right) \varrho\left(Y_{b}\right)$, where "PBW" is used, $\varrho$ is a linear map $\mathbb{R}\left[T_{w}, Y_{b}, w \in W, b \in P\right] \rightarrow \mathbb{R}$ such that $\rho: \mathbb{R}\left[Y^{ \pm 1}\right] \rightarrow \mathbb{R}$ is a (one-dimensional) character and $\varrho\left(T_{w}\right)=$ $\varrho\left(T_{w^{-1}}\right)$. A variant is with $\mathbb{C}$ instead of $\mathbb{R}$. Then $\{\varkappa(H)\}_{\varkappa}^{\varrho}=\{H\}_{\varkappa}^{\varrho}$ by construction and $\{A, B\} \stackrel{\text { def }}{=}\left\{A^{\varkappa} B\right\}_{\varkappa}^{\varrho}=\{B, A\}$.

General problem. Find an integral (analytic) formula for $\{H\} \varrho_{\varkappa}$. It is well defined for any $q, t \in \mathbb{R}^{*}$ (or in $\mathbb{C}^{*}$ ) by construction, but presenting this "algebraic" functional "analytically" is important in DAHA theory (and the key in this paper).

We will stick to the polynomial case through this paper. Namely, $\varrho$ will be the one-dimensional character of affine Hecke algebra $\mathcal{H}_{Y}$ generated by $T_{w}$ and $Y_{b}$, which sends $T_{i} \mapsto t^{1 / 2}, Y_{b} \mapsto t^{(\rho, b)}$ for $i \geq 0, b \in$ $P$. Here $\rho=\frac{1}{2} \sum_{\alpha>0} \alpha$. Generally, the number of different parameters $t$ here equals the number of different lengths $|\alpha|$ in $R$. Then $\{A, B\}$ acts via $\mathcal{X} \times \mathcal{X}$ for the polynomial representation $\mathcal{X}=\mathbb{R}\left[X^{ \pm 1}\right]=\operatorname{Ind}_{\mathcal{H}_{Y}}^{\mathcal{H}}(\varrho)$. 
Generalizing the above definition, level-one anti-involutions $\varkappa$ are such that $\operatorname{dim} \mathcal{H} /\left(\mathcal{J}+\mathcal{J}^{\varkappa}\right)=1$ for $\mathcal{X}=\mathcal{H} / \mathcal{J}, \mathcal{J}=\{H \mid H(1)=0\}$, $1 \in \mathcal{X}$. The Shapovalov ones are obviously level-one. Then $\{H\}_{\varkappa}^{\varrho}$ is defined as the image of $H$ in $\mathcal{H} /\left(\mathcal{J}+\mathcal{J}^{\varkappa}\right)$.

An example. Let $*: g \mapsto g^{-1}$ for $g=X_{a}, Y_{b}, T_{w}, q, t$. It is level-one for generic $q, t$, but obviously not a Shapovalov anti-involution with respect to $Y$. One can prove ${ }^{[2]}$ that there exists the corresponding unique inner product in $\mathcal{X}$ for generic $q, t$ (not for all $t$ if $q$ is generic).

\section{Rational DAHA}

For rational DAHA, the counterpart of $*$ above (serving the "standard" inner product in $\mathcal{X}$ ) is not level-one. The rational DAHA is:

$\mathcal{H}^{\prime \prime} \stackrel{\text { def }}{=}\langle x, y, s\rangle /\left\{[y, x]=\frac{1}{2}+k s, s^{2}=1\right.$, sxs $=-x$, sys $\left.=-y\right\}$.

Accordingly the polynomial representation $\mathcal{X}$ becomes $\mathbb{R}[x]$ with the following action of $\mathcal{H}^{\prime \prime}$ :

$s(x)=-x, \quad x=$ multiplication by $x, y \mapsto D / 2$, where $D=\frac{d}{d x}+\frac{k}{x}(1-s)$ (the Dunkl operator).

Then the anti-involution $x^{*}=x, y^{*}=-y, s^{*}=s$ formally serves the inner product $\int f(x) g(x)|x|^{2 k}$, but it diverges at $\infty$. Algebraically, $\mathbb{R}[x]$ has no $*$-form for $k \notin-1 / 2-\mathbb{Z}_{+}$. Indeed, for $p \in \mathbb{Z}_{+}\left\{1, y\left(x^{p}\right)\right\}=0=$ $\left\{1, c_{p} x^{p-1}\right\}$, where $c_{2 p}=p, c_{2 p+1}=p+1 / 2+k$ (direct from the Dunkl operator). Hence, $\left\{1, x^{p}\right\}=0(\forall p)$ for non-singular $k$ and $\{\}=$,0 .

To fix this problem, let us replace $y$ by $y+x$; then $*$ becomes Shapovalov for such new $y$ (the definition depends on the choice of $y$ ). Indeed, the decomposition $h=\sum c_{a \delta b}\left((y+x)^{*}\right)^{a} s^{\delta}(y+x)^{b}$ exists and is unique $(\delta=0,1)$ for any $h$. Defining the coinvariant by $\{h\} \stackrel{\text { def }}{=}$ $\sum_{\delta=0,1} c_{o \delta o},\{f, g\} \stackrel{\text { def }}{=}\left\{f^{*} g\right\}$, it acts through $\mathbb{R}[x] e^{-x^{2}} \times \mathbb{R}[x] e^{-x^{2}}$ due to $(y+x) e^{-x^{2}}=0$ for the natural action of $\mathcal{H}^{\prime \prime}$ on $e^{-x^{2}}$. Indeed, $\mathbb{R}[x] e^{-x^{2}}$ can be identified with $\mathcal{H}^{\prime \prime} /\left(\mathcal{H}^{\prime \prime}(y+x), \mathcal{H}^{\prime \prime}(s-1)\right)$.

Explicitly, let $p=\frac{a+b}{2}$ for $a, b \in Z_{+}$. Then a direct PBW calculation readily gives that $\left\{x^{a}, x^{b}\right\}=\left(\frac{1}{2}\right)^{p}\left(\frac{1}{2}+k\right) \cdots\left(\frac{1}{2}+k+p-1\right)$. Analytically, we ensured the convergence of $\int_{\mathbb{R}} f g|x|^{2 k}$ via the multiplication of $f$ and $g$ by $e^{-x^{2}}$; let us provide the exact analysis.

The integral presentation for this form is:

$$
\{f, g\}=\frac{1}{i} \int_{-\epsilon+i \mathbb{R}}\left(f g e^{-2 x^{2}}\left(x^{2}\right)^{k}\right) d x /(\cos (\pi k) C),
$$

where $C=\Gamma(k+1 / 2) 2^{k+1 / 2}, \forall k \in \mathbb{C}, \epsilon>0$.

For real $k>-\frac{1}{2}$, one can simply do the following: 


$$
\{f, g\}=\frac{1}{i C} \int_{i \mathbb{R}} f g e^{-2 x^{2}}|x|^{2 k} d x .
$$

Note using $|x|$ here, which is not natural algebraically; one can take here $x^{2 k}$ instead using the technique of hyperspinors (see below).

Let $k=-\frac{1}{2}-m\left(m \in \mathbb{Z}_{+}\right)$. Then we replace $\int_{-\epsilon+i \mathbb{R}} \rightsquigarrow \frac{1}{2}\left(\int_{-\epsilon+i \mathbb{R}}+\int_{\epsilon+i \mathbb{R}}\right)$ and $\{f, g\}$ becomes const $\operatorname{Res}_{0}\left(f g e^{-2 x^{2}} x^{-2 m-1} d x\right)$. The radical of this form is non-zero. It is $\left(x^{2 m+1} e^{-x^{2}}\right)$, which is a unitary $\mathcal{H}^{\prime \prime}$-module with respect to the form $\frac{1}{i} \int_{i \mathbb{R}} f g e^{-2 x^{2}}|x|^{-2 m-1} d x$ restricted to this module (the convergence at $x=0$ is granted). The $*$-form of the quotient $\mathbb{R}[x] /\left(x^{2 m+1}\right)$ is non-positive. See ${ }^{[8]}$ for some details.

\section{General DAHA $\left(A_{1}\right)^{[2]}$}

The $q$-Extended elliptic braid group. It is $\mathcal{B}_{q} \stackrel{\text { def }}{=}$ $\left\langle T, X, Y, q^{1 / 4}\right\rangle /\left\{T X T=X^{-1}, T Y^{-1} T=Y, Y^{-1} X^{-1} Y X T^{2}=q^{-\frac{1}{2}}\right\}$.

Elliptic braid group. It is $\mathcal{B}_{1} \stackrel{\text { def }}{=} \mathcal{B}_{q=1}=\mathcal{B}_{1}=\pi_{1}^{\text {orb }}\left(\{E \backslash 0\} / \mathbf{S}_{2}\right)$, where $E$ is an elliptic curve (a 2-dimensional torus). We will provide below the geometric-topological interpretation of the relations in $\mathcal{B}_{1}$.

DAHA is defined as follows: $\mathcal{H} \stackrel{\text { def }}{=} \mathbb{R}\left[\mathcal{B}_{q}\right] /\left(\left(T-t^{1 / 2}\right)\left(T+t^{-1 / 2}\right)\right)$, where $q=\exp (-1 / a), a>0, t=q^{k}$. Here $k \in \mathbb{R}$ for the positivity questions, but we will need $k \in \mathbb{C}$ when doing analytic continuations.

If $t^{\frac{1}{2}}=1$, then $T^{2}=1$ and we will replace $T$ by $s$. In this case, $\mathcal{H}$ becomes the Weyl algebra extended by $\mathbf{S}_{2}$. I.e. the relations are:

$$
s X s=X^{-1}, s Y s=Y^{-1}, Y^{-1} X^{-1} Y X=q^{-1 / 2}, s^{2}=1 .
$$

Thus DAHA unites Weyl algebras with the Hecke ones. The Heisenberg and Weyl algebras (also called non-commutative tori) are the main tools in quantization of symplectic varieties. So DAHA can be expected to serve "refined quantization" (with extra parameters) of varieties with global or local (in tangent spaces) $W$-structures for Weyl groups $W$.

The whole $P S L_{2}(\mathbf{Z})$ acts projectively in $\mathcal{B}_{q}$ and $\mathcal{H}$ :

$$
\begin{aligned}
& \left(\begin{array}{c}
11 \\
01
\end{array}\right) \sim \tau_{+}: Y \mapsto q^{-1 / 4} X Y, X \mapsto X, T \mapsto T, \\
& \left(\begin{array}{l}
10 \\
11
\end{array}\right) \sim \tau_{-}: X \mapsto q^{1 / 4} Y X, \quad Y \mapsto Y, \quad T \mapsto T .
\end{aligned}
$$

They are directly from topology. The key for us is a pure algebraic fact that $\tau_{+}$is the conjugation by $q^{x^{2}}$, where $X=q^{x}$; use $\mathcal{X}$ below to see this. DAHA FT is for $\tau_{+}^{-1} \tau_{-} \tau_{+}^{-1}=\sigma^{-1}=\tau_{-} \tau_{+}^{-1} \tau_{-}$. 


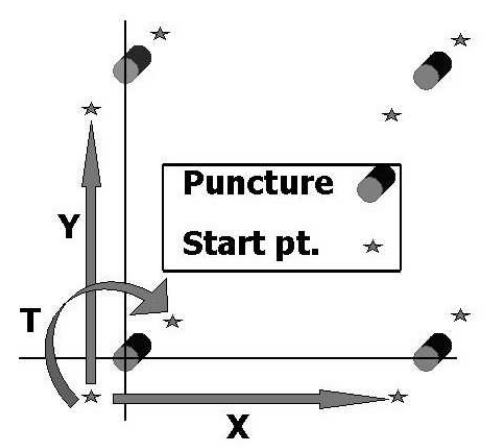

Generators of $\mathcal{B}_{1}$ and relation $Y^{-1} X^{-1} Y X T^{2}=1$

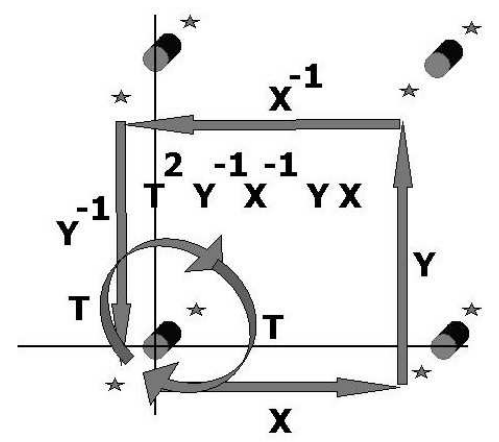

More exactly, the operator Fourier transform is the DAHA automorphism sending: $q^{1 / 2} \mapsto q^{1 / 2}, t^{1 / 2} \mapsto t^{1 / 2}$,

$$
Y \mapsto X^{-1}, X \mapsto T Y^{-1} T^{-1}, T \mapsto T ;
$$

topologically, it is essentially the transposition of the periods of $E$, though it is not an involution; it corresponds to the matrix $\left(\begin{array}{cc}0 & -1 \\ 1 & 0\end{array}\right)$ representing $\sigma^{-1}$.

Polynomial representation. It was defined above as $\operatorname{Ind}_{\mathcal{H}_{Y}}^{\mathcal{H}}(\varrho)$. It is in the space $\mathcal{X}$ of Laurent polynomials of $X=q^{x}$. The action is:

$$
\begin{aligned}
& T \mapsto t^{1 / 2} s+\frac{t^{1 / 2}-t^{-1 / 2}}{q^{2 x}-1}(s-1), Y \mapsto \pi T, \\
& \text { where } \pi=s p, s f(x)=f(-x), s(X)=X^{-1}, \\
& p f(x)=f(x+1 / 2), p(X)=q^{1 / 2} X, t=q^{k} .
\end{aligned}
$$

Here $Y$ becomes the difference Dunkl Operator; $X$ acts by the multiplication. 
The standard AHA stuff (Bernstein's Lemma) gives that $Y+Y^{-1}$ preserves $\mathcal{X}_{\text {sym }} \stackrel{\text { def }}{=}\{$ symmetric (even) Laurent polynomials $\}$; the difference operator $Y+\left.Y^{-1}\right|_{\text {sym }}$ is sometimes called the $q, t$-radial part.

Basic inner products. ${ }^{[2]}$ Note that we do not conjugate $q, t$ below (a simplest way to supply $\mathcal{X}$ with an inner product).

For $X=q^{x}, q=\exp \left(-\frac{1}{a}\right)$ and the Macdonald truncated $\theta$-function

$$
\begin{gathered}
\mu(x)=\prod_{i=0}^{\infty} \frac{\left(1-q^{i+2 x}\right)\left(1-q^{i+1-2 x}\right)}{\left(1-q^{i+k+2 x}\right)\left(1-q^{i+k+1-2 x}\right)}, \text { we set: } \\
\langle f, g\rangle_{1 / 4} \stackrel{\text { def }}{=} \frac{1}{2 \pi a i} \int_{1 / 4+P} f(x) T(g)(x) \mu(x) d x, \text { where } P=[-\pi i a, \pi i a] .
\end{gathered}
$$

Theorem 5.1. For $k>-\frac{1}{2}$ (generally, $\Re k>-\frac{1}{2}$ ), $\langle f, g\rangle_{1 / 4}=(f T(g) \mu)_{\mathrm{CT}}$. The later inner product in $\mathcal{X}$ serves for any $k$ the anti-involution $\diamond$ : $T^{\diamond}=T, Y^{\diamond}=Y, X^{\diamond}=T^{-1} X T$. The inner product $\langle f, g\rangle_{1 / 4}$ does it only for $k>-1 / 2$, where it is positive definite in $\mathcal{X}=\mathbb{R}\left[X^{ \pm 1}\right]$; however it remains symmetric for any $k$.

Proof. The coincidence of two formulas for $\Re k>-1 / 2$ and the fact that $\diamond$ serves $(f T(g) \mu)_{\mathrm{CT}}$ are from ${ }^{[2]}$ (for any root systems). The positivity is straightforward via the norm-formulas for $E$-polynomials; let us provide a directly proof using that $\pi(\mu)=\mu(1 / 2-x)=\mu$.

(a) The $E$-polynomials are defined as follows: $Y\left(E_{n}\right)=q^{-n_{\sharp}} E_{n}$, where $n_{\sharp}=\frac{n-k}{2}$ as $n \leq 0, n_{\sharp}=\frac{n+k}{2}$ as $n>0, E_{n}=X^{n}+$ (lower terms). Here $X_{m}$ is lower than $X_{n}$ if either $|m|<|n|$ or $m=-n>0$.

(b) Then $\left\langle E_{n}, E_{m}\right\rangle_{1 / 4}=C_{n} \delta_{n m}$ for some constant $C_{n}$ due to $Y^{\diamond}=Y$.

(c) One has: $C_{n}=q^{-n_{\sharp}} \frac{1}{i} \int_{1 / 4+P} E_{n} \overline{E_{n}} \mu(x) d x>0$, since $\pi(x)=\bar{x}$ (the latter is complex conjugation) and $\mu(x)>0$ at $1 / 4+P$; use that $T\left(E_{n}\right)=\pi Y\left(E_{n}\right)=q^{-n_{\sharp}} \pi\left(E_{n}\right)=q^{-n_{\sharp}} \overline{E_{n}}$.

Imaginary Integration. For $\Re k>-\frac{1}{2}, f, g \in \mathcal{X}$, we set

$$
\begin{aligned}
\langle f, g\rangle_{1 / 4}^{\gamma, \infty} \stackrel{\text { def }}{=} \frac{1}{i} \int_{\frac{1}{4}+i \mathbb{R}} f T(g) q^{-x^{2}} \mu(x) d x & \\
& =\frac{1}{2 i \sqrt{\pi a}} \int_{\frac{1}{4}+P} f T(g) \sum_{j=-\infty}^{\infty} q^{j^{2} / 4+j x} \mu(x) d x,
\end{aligned}
$$

where we use that $f, T(g)$ and $\mu$ are $P$-periodic and that $q^{-x^{2}}$ is such with a multiplier. Then we employ the functional equation for the theta-function; see Section 2.2.2 from ${ }^{[2]}$. For such $k$, this inner product is symmetric and positive (as $k>-1 / 2$ ); it serves the anti-involution

$$
\varkappa: \quad T^{\varkappa}=T, X^{\varkappa}=T^{-1} X T, Y^{\varkappa}=q^{-1 / 4} X Y .
$$

The latter involution is $\diamond$ above conjugated by $\tau_{+}$, which reflects the multiplication the integrand by $q^{-x^{2}}$. So the relation to $\varkappa$ (only for $\Re k>-1 / 2$ ) and the positivity follow from Theorem 5.1. 


\section{Analytic Continuation}

The ingredients are as follows: the Shapovalov $\varkappa$ above (for $Y$ ) and the standard coinvariant $\varrho($ serving $\mathcal{X})$. Recall that

$$
\varrho\left(\sum_{a, b \in \mathbb{Z}}^{\epsilon=0,1} c_{a \epsilon b}\left(Y^{\varkappa}\right)^{a} T^{\epsilon} Y^{b}\right) \stackrel{\text { def }}{=} \sum c_{a \epsilon b} t^{\frac{a+\epsilon+b}{2}}
$$

and the corresponding form is

$$
\{A, B\}_{\varkappa}^{\varrho} \stackrel{\text { def }}{=} \varrho\left(A^{\varkappa} B\right)=\{B, A\}_{\varkappa}^{\varrho} \text { in } \mathcal{H} \ni A, B .
$$

The latter acts via $\mathcal{X} \times \mathcal{X}, \mathcal{X}=\mathbb{R}\left[X^{ \pm 1}\right]$, and satisfies the normalization $\{1,1\}=1$ by construction. This form is regular (analytic) for all $k \in \mathbb{C}$.

Theorem 6.1. For $\Re k>-1 / 2$, one has: $G(k)\{f, g\}_{\varkappa}^{\varrho}=\langle f, g\rangle_{1 / 4}^{\gamma, \infty}$, where $G(k)=\sqrt{\pi a} \prod_{j=1}^{\infty} \frac{1-q^{k+j}}{1-q^{2 k+j}} \quad$ (the latter is from ${ }^{[2]}$, Theorem 2.2.1).

Proof. Let $\Phi_{\epsilon}^{k}(f, g) \stackrel{\text { def }}{=} \frac{1}{i} \int_{\epsilon+i \mathbb{R}} f T(g) q^{-x^{2}} \mu(x) d x$ for the path $\mathcal{C} \stackrel{\text { def }}{=}$ $\{\epsilon+i \mathbb{R}\}$. For such a path, bad (singular) $k$ are $\left\{2 \mathcal{C}-1-\mathbb{Z}_{+},-2 \mathcal{C}-\mathbb{Z}_{+}\right\}$ (when poles of $\mu$ belong to $\mathcal{C}$ ); so $\{\Re k>-1 / 2\}$ are all good as $\epsilon=1 / 4$. Then we use that the theorem holds for $\Re k \gg 0$.

The case $\epsilon=0$. Then $\Phi_{0}^{k}(f, g)$ coincides with $G(k)\{f, g\} \stackrel{\varrho}{\varkappa}$ only for $\Re k>0$. For any $k$, this form is symmetric and its anti-involution sends $T \mapsto T, X \mapsto X^{\varkappa}=T^{-1} X T$ (the image of $Y$ is not $Y^{\varkappa}$ if $\Re k<0$ ).

Comparing $\epsilon=0$ and $\epsilon=\frac{1}{4}$ for $0>\Re k>-\frac{1}{2}$. (This is actually the induction step for the analytic continuation to any negative $\Re k$ ).

Let us assume that $F=f T(g) \in \mathbb{R}\left[X^{ \pm 2}\right]$. We mainly follow ${ }^{[11]}$, Section 2.2. By picking up the residues between the $\mathcal{C}$-paths at 0 and at $1 / 4$, one obtains that $\Phi_{\frac{1}{4}}^{k}=\Phi_{0}^{k}+A\left(-\frac{k}{2}\right) \mu^{\bullet}\left(-\frac{k}{2}\right) F\left(-\frac{k}{2}\right) \stackrel{\text { def }}{=} \widehat{\Phi}^{k}$, where $A(\widetilde{k})=\sqrt{\pi a} \sum_{m=-\infty}^{\infty} q^{m^{2}+2 m \widetilde{k}}$ (the contribution of $q^{-x^{2}}$ ), and

$$
F\left(-\frac{k}{2}\right)=f T(g)\left(x \mapsto-\frac{k}{2}\right), \quad \mu^{\bullet}\left(-\frac{k}{2}\right)=\prod_{j=0}^{\infty} \frac{\left(1-q^{k+j+1}\right)\left(1-q^{-k+j}\right)}{\left(1-q^{1+j}\right)\left(1-q^{2 k+j+1}\right)}=
$$
$\left(\left(1-q^{2 x+k}\right) \mu(x)\right)(x \mapsto-k / 2)$.

Importantly, $\widehat{\Phi}^{k}$ is meromorphic for $\Re k>-1$ (i.e. beyond $-\frac{1}{2}$ for $\Phi_{\frac{1}{4}}^{k}$ ); so it coincides with $G(k)\{f, g\}_{\varkappa}^{\varrho}$ there. We note that $\widehat{\Phi}^{k}$ is symmetric for any $k$. Indeed: $f T(g)\left(-\frac{k}{2}\right)=t^{1 / 2} f g\left(-\frac{k}{2}\right)=T(f) g\left(-\frac{k}{2}\right)$ due to $T=\frac{q^{2 x+k / 2}-q^{-k / 2}}{q^{2 x}-1} s-\frac{q^{k / 2}-q^{-k / 2}}{q^{2 x}-1}$ and $\left(q^{2 x+k / 2}-q^{-k / 2}\right)(x \mapsto-k / 2)=0$.

Main Theorem 6.2. For $F=f T(g) \in \mathbb{R}\left[X^{ \pm 2}\right]$ and for any $\Re k<0$ : $G(k)\{f, g\}_{\varkappa}^{\varrho}=\Phi_{0}^{k}+\mu^{\bullet}(-k / 2) \sum_{\widetilde{k} \in \widetilde{K}} A(\widetilde{k})\left(\mu^{\bullet}(\widetilde{k}) / \mu^{\bullet}\left(-\frac{k}{2}\right)\right) F(\widetilde{k})$, for $\widetilde{K}=\left\{n_{\sharp},|n| \leq m\right\}=\{-k / 2\} \cup\left\{ \pm \frac{k+j}{2}, 1 \leq j \leq m\right\}, m \stackrel{\text { def }}{=}[\Re(-k)]$, 
where $[\cdot]=$ integer part, and $\mu^{\bullet}\left( \pm \frac{k+j}{2}\right) / \mu^{\bullet}\left(-\frac{k}{2}\right)=t^{-j_{ \pm}} \prod_{i=1}^{j_{ \pm}} \frac{1-t^{2} q^{i}}{1-q^{i}}$ for $j_{+}=j-1, j_{-}=j$. Generally, if $F \in \mathbb{R}\left[X^{ \pm 1}\right]$ (not in $\mathbb{R}\left[X^{ \pm 2}\right]$ as above), the poles of $\mu$ are given by the relations $q^{-\frac{1}{2}} X \in \pm q^{\mathbb{Z}_{+} / 2} t^{\frac{1}{2}} \ni X^{-1}$, and the summation must be "doubled" accordingly.

Here we count "jumps" through the walls $\Re k=-j \in-\mathbb{Z}_{+}$. The duplication of the summation for $F \in \mathbb{R}\left[X^{ \pm 1}\right]$ corresponds to the passage from affine Weyl group $\widetilde{W}$ to its extension $\widehat{W}$ by $\Pi=\mathbb{Z}_{2}$ in the $p$-adic limit $q \rightarrow 0, X \mapsto Y$ (discussed below).

Importantly, here and for any root systems only the total sum is an $\mathcal{H} \mathcal{H}$-invariant form. The partial sums with respect to the dimensions of the integration domains are symmetric and even $\mathcal{H}_{X}$-invariant, but they are not $\mathcal{H} \mathcal{H}$-invariant.

Corollary 6.3. The form $\{f, g\}_{\varkappa}^{\varrho}$ is degenerate exactly at the poles of $G(k): k=-\frac{1}{2}-m, m \in \mathbb{Z}_{+}$. For such $k$, the quotient of $\mathcal{X}$ by its radical is a direct sum of 2 irreducible $\mathcal{H} \mathfrak{H}$-modules of dim $=2 m+1$ ("perfect" in the terminology from $\left.{ }^{[2]}\right)$, transposed by the map $X \mapsto-X$.

The radical here is the ideal $\left(E_{2 m+1}\right)$, which is a unitary $\mathcal{H} H$-module with respect to $\Phi_{0}^{k}$, matching the analogous fact for the rational DAHA $\mathcal{H U}^{\prime \prime}$ observed above. The rational limit is as follows: $q=e^{\hbar}, t=$ $q^{k}, Y=e^{-\sqrt{\hbar} \bar{y}}, X=e^{\sqrt{\hbar} \bar{x}}, \hbar \rightarrow 0\left(\bar{x}\right.$ is not $x$ from $\left.X=q^{x}\right)$. One has $q^{x^{2}}=e^{\bar{x}^{2}}$, and $\mu(x)$ becomes essentially $\bar{x}^{2 k}$ under this limit. So the space Funct $(\widetilde{K})$ (upon the restriction to $F \in \mathbb{R}\left[X^{ \pm 2}\right]$, which does not influence the rational limit) directly maps to the $\mathcal{H U}^{\prime \prime}$-module $\mathbb{R}[\bar{x}] /\left(\bar{x}^{2 m+1}\right.$ ) (in terms of $\bar{x}, \bar{y}$ ).

Theorem 6.4. For any root system $R \subset \mathbb{R}^{n}$, a Shapovalov or levelone anti-involution $\varkappa$, and for the coinvariant $\varrho$ serving the polynomial representation, the corresponding DAHA-invariant form can be represented as a finite sum of integrals over translations of $\imath \mathbb{A}$ for proper subspaces $\mathbb{A} \subset \mathbb{R}^{n}$, called double-affine $q$,t-residual subtori, starting with the full imaginary integration.

The program is to $(a)$ find (explicitly) these subtori for any root systems, (b) calculate the corresponding $C$-coefficients (the $q$-deformation of the AHA Plancherel measure), and finally $(c)$ perform the $p$-adic limit $(q \rightarrow 0)$, which steps are technically non-trivial (even in type $A$ ).

\section{P-ADIC LIMIT}

For the AHA $\mathcal{H}$ of type $A_{1}$, we set $s=s_{1}, \omega=\omega_{1}, \pi=s \omega$. Let

$$
\psi_{n} \stackrel{\text { def }}{=} t^{-\frac{|n|}{2}} T_{n \omega} \mathcal{P}_{+}, \mathcal{P}_{+}=\left(1+t^{1 / 2} T\right) /(1+t) \text { for } n \in \mathbb{Z} .
$$


One can naturally consider them as polynomials in terms of $Y \stackrel{\text { def }}{=}$ $T_{\omega}=\pi T$; then they become the Matsumoto spherical functions. This identification is based on the analysis by Opdam ${ }^{[22]}$ and the author ${ }^{[11]}$; see also ${ }^{[2]}$ (Section 2.11.2) and ${ }^{[16,8]}$. Accordingly, the SatakeMacdonald $p$-adic spherical functions become $\mathcal{P}_{+} \psi_{n}(n \geq 0)$.

Theorem 7.1. For $n \in \mathbb{Z}$, the polynomials $E_{n}(X) / E_{n}\left(t^{-\frac{1}{2}}\right)$ become $\psi_{n}$ as $q \rightarrow 0$ upon the following substitution:

$$
f(X) \mapsto f(X)^{\prime} \stackrel{\text { def }}{=} f\left(X \mapsto X^{\prime}=Y, t \mapsto t^{\prime}=\frac{1}{t}\right) .
$$

Let $\mu_{0}=\mu(q \rightarrow 0)=\frac{1-X}{1-t X},\{f, g\}_{0}=\left(f T(g) \mu_{0}\right)_{\mathrm{CT}}$. Then for $\left\langle T_{\widehat{w}}\right\rangle=\delta_{i d, \widehat{w}}$ and the standard anti-involution $T_{\widehat{w}}^{\star}=T_{\widehat{w}^{-1}}$ in $\mathcal{H}$, one has:

$$
\{f, g\}_{0}\left(t \mapsto t^{\prime}\right)=\left(t^{1 / 2}+t^{-1 / 2}\right)\left\langle\left(f^{\prime} \mathcal{P}_{+}\right)\left(g^{\prime} \mathcal{P}_{+}\right)^{\star}\right\rangle \text { for } f, g \in \mathcal{X},
$$

which is actually the nonsymmetric AHA Plancherel formula for the $p$-adic Fourier transform. Here $t^{\prime}, f^{\prime}, g^{\prime}$ are as above.

The corresponding version of the Main Theorem (compatible with the $p$-adic limit) is as follows. The Gaussian collapses and we must omit it and use the integration over the period instead of the imaginary integration. We continue using the notations $j_{ \pm}=\{j-1, j\}, t=q^{k}$.

Theorem 7.2. For $q=e^{-\frac{1}{a}}, M \in \mathbb{N} / 2, F(x)=f T(g)\left(q^{x}\right) \in \mathbb{R}\left[q^{ \pm 2 x}\right]$ :

$$
\begin{aligned}
& (F \mu)_{\mathrm{CT}}=\frac{1}{2 \pi a M \imath} \int_{-\pi a M \imath}^{+\pi a M \imath} F(x) \mu(x) d x \\
& +\mu^{\bullet}\left(-\frac{k}{2}\right) \times\left(F\left(-\frac{k}{2}\right)+\sum_{j=1, \pm}^{[\Re(-k)]} F\left( \pm \frac{k+j}{2}\right) t^{-j_{ \pm}} \prod_{i=1}^{j_{ \pm}} \frac{1-t^{2} q^{i}}{1-q^{i}}\right) .
\end{aligned}
$$

Here $k$ is arbitrary. The left-hand side is entirely algebraic and meromorphic for any $k$ by construction. Namely ${ }^{[2]},(F \mu)_{\mathrm{CT}}=(\mu)_{\mathrm{CT}}\left(F \mu^{\circ}\right)_{\mathrm{CT}}$,

$$
\mu^{\circ} \stackrel{\text { def }}{=} \mu(x) /(\mu)_{\mathrm{CT}}=1+\frac{q^{k}-1}{1-q^{k+1}}\left(q^{2 x}+q^{1-2 x}\right)+\cdots
$$

is a series in terms of $\left(q^{2 m x}+q^{m-2 m x}\right)$ for $m \geq 0$ with rational $q$, $t$ coefficients, which is essentially Ramanujan's ${ }_{1} \Psi_{1}$-summation, and

$$
(\mu)_{\mathrm{CT}}=\frac{\left(1-q^{k+1}\right)^{2}\left(1-q^{k+2}\right)^{2} \cdots}{\left(1-q^{2 k+1}\right)\left(1-q^{2 k+2}\right) \cdots(1-q)\left(1-q^{2}\right) \cdots} .
$$

One can replacing the integral above with the corresponding sum of the residues, which is an interesting generalization of the classical formula for the reciprocal of the theta-function ${ }^{[1]}$. Its extension to any root systems requires Jackson integrations; see Section 3.5 from ${ }^{[2]}$. 
Proposition 7.3. For $\Re k<-m \in-\mathbb{Z}_{+}$and $F(x) \in q^{-2 m} \mathbb{R}\left[q^{+2 x}\right]$,

$$
\begin{aligned}
& \frac{1}{\pi a \imath} \int_{-\pi a \imath / 2}^{+\pi a \imath / 2} F(x) \mu(x) d x= \\
& \mu^{\bullet}\left(-\frac{k}{2}\right) \times\left(-F\left(-\frac{k}{2}\right)-\sum_{j=1}^{[\Re(-k)]} F\left(-\frac{k+j}{2}\right) t^{-j} \prod_{i=1}^{j} \frac{1-t^{2} q^{i}}{1-q^{i}}\right. \\
&\left.+\sum_{j=[\Re(-k)]+1}^{\infty} F\left(\frac{k+j}{2}\right) t^{1-j} \prod_{i=1}^{j-1} \frac{1-t^{2} q^{i}}{1-q^{i}}\right), \\
&(F \mu)_{\mathrm{CT}}=\mu^{\bullet}\left(-\frac{k}{2}\right) \times\left(\sum_{j=1}^{\infty} F\left(\frac{k+j}{2}\right) t^{1-j} \prod_{i=1}^{j-1} \frac{1-t^{2} q^{i}}{1-q^{i}}\right) .
\end{aligned}
$$

Switching in (7.2) to $X=q^{x}$ and making $a=\frac{1}{M}$ for $M \rightarrow \infty$ (then $q \rightarrow 0)$, let $k=-c a$ for $c>0$. Then $t=e^{-\frac{k}{a}} \rightarrow e^{c}$ and the formula above under $\Re k \rightarrow 0_{-}$becomes the Heckman-Opdam one; recall that DAHA with $t>1$ is related to AHA from Section 2 for $t^{\prime}=\frac{1}{t}<1$. Here and for any root systems, only $A H A$ residual subtori contribute for $M \gg 0$.

\section{Conclusion}

Let us summarize the main elements and steps of the construction we propose. The ingredients are as follows.

(a) Shapovalov anti-involution $\varkappa$ of $\mathcal{H} \mathcal{H}$ (with respect to the subalgebra $\left.\mathcal{Y}=\mathbb{R}\left[Y_{b}\right]\right)$, i.e. such that $\left\{\varkappa\left(Y_{a}\right) T_{w} Y_{b}\right\}$ form a $(\mathrm{PBW})$ basis of $\mathcal{H}$;

(b) the corresponding coinvariant: $\varrho: \mathcal{H H} \rightarrow \mathbb{R}$ satisfying $\varrho(\varkappa(H))=$ $\varrho(H)$ (for any character of $\mathcal{Y}$ and $\varrho$ on $\mathbf{H}$ s.t. $\varrho\left(T_{w}-T_{w^{-1}}\right)=0$ );

(c) the corresponding Shapovalov form $\{f, g\} \stackrel{\text { def }}{=} \varrho\left(A^{\varkappa} B\right)$ for $A, B \in$ $\mathcal{H H}$, satisfying $\{1,1\}=1$ and analytic for any $k$.

The main problem is to express $\{f, g\}_{\varkappa}^{\varrho}$ as a sum of integrals over the DAHA residual subtori for any (negative) $\Re k$. Then one can try to generalize this formula to arbitrary DAHA anti-involutions (any "levels") and any induced modules.

Hyperspinors ${ }^{[7,8,10,20]}$. An important particular case of the program above is a generalization of the integral formulas from the spherical case to the whole regular representation of $A H A$. The technique of hyperspinors is expected to be useful here; they were called $W$-spinors in prior works ( $W$ stands for the Weyl group).

The $W$-spinors are simply collections $\left\{f_{w}, w \in W\right\}$ of elements $f_{w} \in$ $A$ with a natural action of $W$ on the indices. If $A$ (an algebra or a sheaf of algebras) has its own (inner) action of $W$ and $f_{w}=w^{-1}\left(f_{i d}\right)$, they are called principle spinors. Geometrically, hyperspinors are $\mathbb{C} W$ valued functions on any manifolds, which is especially interesting for 
those with an action of $W$. The technigue of spinors can be seen as a direct generalization of supermathematics, which is the case of the root system $A_{1}$, from $W=\mathbf{S}_{2}$ to arbitrary Weyl groups.

For instance, Laurent polynomials with the coefficients in the group algebra $\mathbb{C} W$ are considered instead of $\mathcal{X}$, the integration is defined upon the projection $W \ni w \mapsto 1$ (a counterpart of taking the even part of a super-function), and so on and so forth. No "brand new" definitions are necessary here, but the theory quickly becomes involved.

The $W$-spinors proved to be very useful for quite a few projects. One of the first instances was the author's proof in ${ }^{[7]}$ of the CherednikMatsuo theorem, an isomorphism between the $A K Z$ and $Q M B P$. An entirely algebraic version of this argument was presented in ${ }^{[20]}$; also see ${ }^{[2]}$. This proof included the concept of the fundamental group for the configuration space associated with $W$ or its affine analogs without fixing a starting point, à la Grothendieck. A certain system of cutoffs and the related complex hyperspinors can be used instead. The corresponding representations of the braid group becomes a 1-cocycle on $W$ (a much more algebraic object then the usual monodromy).

A convincing application of the technique of hyperspinors was the theory of non-symmetric $q$-Whittaker functions. The Dunkl operators in the theory of Whittaker functions (which are non-symmetric as well as the corresponding Toda operators) simply cannot be defined without hyperspinors and the calculations with them require quite a mature level of the corresponding technique. See ${ }^{[8]}$ and especially ${ }^{[10]}$ (the case of arbitrary root systems). The Harish-Chandra-type decomposition formula for global nonsymmetric functions from ${ }^{[6]}$ (for $A_{1}$ ) is another important application; hyperspinors are essential here.

By the way, $x^{2 k}$ for complex $k$, which is one of the key in the rational theory (see above), is a typical complex spinor, i.e. a collection of two (independent) branches of this function in the upper and lower half-planes. To give another (related) example, the Dunkl eigenvalue problem always has $|W|$ independent spinor solutions; generally, only one of them is a function. In the case of $\mathcal{H}^{\prime \prime}$ for $A_{1}$ (above), both fundamental spinor solutions for singular $k=-1 / 2-m, m \in \mathbb{Z}_{+}$are functions. See ${ }^{[8]}$ for some details.

A natural question is, do we have hypersymmetric physics theories for any Weyl groups $W$, say " $W$-hypersymmetric Yang-Mills theory"?

Jantzen filtration. It is generally a filtration of the polynomial representation of $\mathcal{X}$ in terms of AHA modules, not DAHA modules, for $\Re k<0$. The top module is the quotient of $\mathcal{X}$ by the radical of the sum of integral terms for the smallest residual subtori (points in many 
cases). Then we restrict the remaining sum to this radical and continue by induction with respect to the dimension of the (remaining) subtori.

Sometimes certain sums for residual subtori of dimensions smaller than $n$ are DAHA-invariant; then $\mathcal{X}$ is reducible. We expect that the reducibility of $\mathcal{X}$ always can be seen this way, which includes the degenerations of DAHA. For $A_{n}$, the corresponding Jantzen filtration provides the whole decomposition of $\mathcal{X}$ in terms of irreducible DAHA modules, the so-called Kasatani decomposition ${ }^{[13,14]}$. Generally, the corresponding quotients can be DAHA-reducible.

For instance, the bottom module of the Jantzen filtration has the inner product that is (the restriction of) the integration over the whole $i \mathbb{R}^{n}$. This provides some a priori way to analyze its signature (positivity), which is of obvious interest. The bottom DAHA submodule of $\mathcal{X}$ was defined algebraically (without the Jantzen filtration) in ${ }^{[4]}$. Indeed, it appeared semisimple under certain technical restrictions. For $A_{n}$, this is related to the so-called wheel conditions.

Let us discuss a bit the rational case. The form $\{f, g\} \underset{\varkappa}{\varrho}$ for $\mathcal{H}^{\prime \prime}$ can be expected to have a presentations in terms of integrals over the $x$-domains with $\Re x$ in the boundary of a tube neighborhood of the resolution of the cross $\prod_{\alpha \in R_{+}}(x, \alpha)=0$ over $\mathbb{R}$. The simplest example is the integration over $\pm i \epsilon+\mathbb{R}$ for $A_{1}$. This resolution (presentation of the cross as a divisor with normal crossings) is due to the author (Publ. of RIMS, 1991), de Concini - Procesi, and Beilinson - Ginzburg.

This can be used to study the bottom module of the polynomial representation for singular $k_{o}=-\frac{s}{d_{i}}$, assuming that it is well-defined and $\mathcal{H} \mathcal{H}^{\prime \prime}$-invariant. When $s=1$ (not for any $s$ ), it can be proved unitary in some interesting cases; see Etingof et al. in the case of $A_{n}{ }^{[14]}$. The restriction of the initial (full) integration over $\mathbb{R}^{n}$ provides a natural approach to this phenomenon. See Section 4 in the case of $A_{1}$.

The DAHA-decomposition of the polynomial or other modules is a natural application of the integral formulas for DAHA-invariant forms, but we think that knowing such formulas is necessary for the DAHA harmonic analysis even if the corresponding modules are irreducible.

Acknowledgements. The author thanks RIMS, Kyoto university for the invitation, and the participants of his course at UNC. Many thanks to the referee for important remarks.

\section{REFERENCES}

[1] L. Carlitz, A finite analog of the reciprocal of a theta function, Pubblications de la Faculté D'électrotechnique De L'Université À Belgrade. Ser. Math. et Phys. 412-460 (1973), 97-99.

[2] I. Cherednik, Double affine Hecke algebras, London Mathematical Society Lecture Note Series, 319, Cambridge University Press, 2006. 
[3] — Difference Macdonald-Mehta conjecture, IMRN 10 (1997), 449467.

[4] _ Nonsemisimple Macdonald polynomials, Selecta Math. 14: 3-4 (2009), 427-569.

[5] Whittaker limits of difference spherical functions, IMRN, 20 (2009), 3793-3842.

[6] _ On Harish-Chandra theory of global nonsymmetric functions, arXiv:1407.5260 (2014).

[7] _ Integration of quantum many-body problems by affine Knizhnik-Zamolodchikov equations, preprint RIMS 776 (1991), Adv. Math. 106 (1994), $65-95$.

[8] — X. Ma, Spherical and Whittaker functions via DAHA I,II, Selecta Mathematica (N.S.), 19:3 (2013), 737-817, 819-864.

[9] — D. Orr, One-dimensional nil-DAHA and Whittaker functions I, Transformation Groups 17:4 (2012), 953-987; arXiv:math/0111130v1 (2011).

[10] _ Nonsymmetric difference Whittaker functions, Mathematische Zeitschrift 279:3 (2015), 879-938.

[11] _ and V. Ostrik, From Double Hecke Algebras to Fourier Transform, Selecta Math., New Ser. 8 (2003), 1-89; arXiv:math/0111130.

[12] D. Ciubotaru, M. Kato, S. Kato, On characters and formal degrees of discrete series of affine Hecke algebras of classical types, Inventiones mathematicae, 187:3 (2012), 589-635.

[13] N. Enomoto, Composition factors of polynomial representation of DAHA and crystallized decomposition numbers, J. Math. Kyoto Univ. 49:3 (2009), 441-473.

[14] P. Etingof, E. Stoica, with an appendix by S. Griffeth, Unitary representations of rational Cherednik algebras, Represent. Theory 13, (2009), 349-370.

[15] G.J. Heckman, and E.M. Opdam, Harmonic analysis for affine Hecke algebras, Current Developments in Mathematics (S.-T. Yau, editor), Intern. Press, Boston (1996).

[16] B. Ion, Nonsymmetric Macdonald polynomials and matrix coefficients for unramified principal series, Advances in Mathematics 201 (2006), 36-62.

[17] D. Kazhdan, G. Lusztig, Proof of the Deligne-Langlands conjecture for Hecke algebras, Inventiones Math. 87 (1987), 153-215.

[18] G. Lusztig, Green functions and character sheaves, Ann. Math. 131 (1990), $355-408$.

[19] J. Stokman, The c-function expansion of a basic hypergeometric function associated to root systems, Ann. Math. 179:1 (2014), 253-299.

[20] E. Opdam, Harmonic analysis for certain representations of graded Hecke algebras, Acta Math. 175 (1995), 75-121.

[21] _ Hecke algebras and harmonic analysis, in: Proceedings of the International Congress of Mathematicians - Madrid II, 1227-1259, EMS Publ. House, 2006.

[22] _ A generating formula for the trace of the Iwahori-Hecke algebra, Progress in Mathematics 210 (2003), 301-323; arXiv:math/0101006.

[23] _ M. Solleveld, Discrete series characters for affine Hecke algebras and their formal degrees, Acta Math. 205:1 (2010), 105-187.

(I. Cherednik) Department of Mathematics, UnC Chapel Hill, North CAROLINA 27599, USA, CHEREd@MATH.UNC.EDU 